\title{
Epigenetic prediction of major depressive disorder
}

\author{
Miruna C. Barbu ${ }^{1} \cdot$ Xueyi Shen ${ }^{1} \cdot$ Rosie M. Walker $\mathbb{D}^{2,3} \cdot$ David M. Howard $\mathbb{D}^{1,4} \cdot$ Kathryn L. Evans $\mathbb{D}^{2,3} \cdot$ \\ Heather C. Whalley $\mathbb{1}^{1} \cdot$ David J. Porteous $\mathbb{D}^{2,3} \cdot$ Stewart W. Morris ${ }^{2,3} \cdot$ lan J. Deary $\mathbb{B}^{3} \cdot$ Yanni Zeng ${ }^{5,6}$. \\ Riccardo E. Marioni ${ }^{2,3} \cdot$ Toni-Kim Clarke ${ }^{1} \cdot$ Andrew M. Mclntosh $\left(^{1,3}\right.$
}

Received: 15 November 2019 / Revised: 21 May 2020 / Accepted: 1 June 2020 / Published online: 10 June 2020

(c) The Author(s) 2020. This article is published with open access

\begin{abstract}
Variation in DNA methylation (DNAm) is associated with lifestyle factors such as smoking and body mass index (BMI) but there has been little research exploring its ability to identify individuals with major depressive disorder (MDD). Using penalised regression on genome-wide CpG methylation, we tested whether DNAm risk scores (MRS), trained on 1223 MDD cases and 1824 controls, could discriminate between cases $(n=363)$ and controls $(n=1417)$ in an independent sample, comparing their predictive accuracy to polygenic risk scores (PRS). The MRS explained $1.75 \%$ of the variance in MDD $\left(\beta=0.338, p=1.17 \times 10^{-7}\right)$ and remained associated after adjustment for lifestyle factors $\left(\beta=0.219, p=0.001, R^{2}=\right.$ $0.68 \%)$. When modelled alongside PRS $\left(\beta=0.384, p=4.69 \times 10^{-9}\right)$ the MRS remained associated with MDD $(\beta=0.327$, $p=5.66 \times 10^{-7}$ ). The MRS was also associated with incident cases of MDD who were well at recruitment but went on to develop MDD at a later assessment $\left(\beta=0.193, p=0.016, R^{2}=0.52 \%\right)$. Heritability analyses found additive genetic effects explained $22 \%$ of variance in the MRS, with a further $19 \%$ explained by pedigree-associated genetic effects and $16 \%$ by the shared couple environment. Smoking status was also strongly associated with MRS $\left(\beta=0.440, p \leq 2 \times 10^{-16}\right)$. After removing smokers from the training set, the MRS strongly associated with BMI $(\beta=0.053, p=0.021)$. We tested the association of MRS with 61 behavioural phenotypes and found that whilst PRS were associated with psychosocial and mental health phenotypes, MRS were more strongly associated with lifestyle and sociodemographic factors. DNAm-based risk scores of MDD significantly discriminated MDD cases from controls in an independent dataset and may represent an archive of exposures to lifestyle factors that are relevant to the prediction of MDD.
\end{abstract}

These authors contributed equally: Toni-Kim Clarke, Andrew M. McIntosh

Supplementary information The online version of this article (https:// doi.org/10.1038/s41380-020-0808-3) contains supplementary material, which is available to authorized users.

Andrew M. McIntosh

andrew.mcintosh@ed.ac.uk

1 Division of Psychiatry, Centre for Clinical Brain Sciences, University of Edinburgh, Edinburgh, UK

2 Centre for Genomic and Experimental Medicine, Institute of Genetics and Molecular Medicine, University of Edinburgh, Edinburgh, UK

3 Centre for Cognitive Ageing and Cognitive Epidemiology, School of Philosophy, Psychology and Language Sciences, University of Edinburgh, Edinburgh, UK

\section{Introduction}

Major depressive disorder (MDD) is a disabling condition with an estimated point prevalence of $4.4 \%$ [1]. Recent genome-wide association studies (GWASs) have begun to elucidate the genetic architecture of MDD [2,3] and polygenic risk scores (PRS) derived from the most recent study of 246,363 depression cases and 561,190 controls explain 1.5-3.2\% of MDD risk in independent cohorts [4]. As sole

4 Social Genetic and Developmental Psychiatry Centre, Institute of Psychiatry, Psychology \& Neuroscience, King's College London, London, UK

5 Faculty of Forensic Medicine, Zhongshan School of Medicine, Sun Yat-Sen University, 74 Zhongshan 2nd Road, Guangzhou 510080, China

6 Guangdong Province Translational Forensic Medicine Engineering Technology Research Center Zhongshan School of Medicine, Sun Yat-Sen University, 74 Zhongshan 2nd Road, Guangzhou, China 
predictors of MDD status, PRS currently have limited clinical utility and may not capture the larger environmental contributions to risk.

Variation in DNA methylation (DNAm) is affected by both genetic and environmental factors, which act in combination to confer risk for diseases and complex traits [5]. DNAm has recently been studied in relation to MDD [6, 7]. An epigenome-wide association study (EWAS) of 7948 European individuals identified $3 \mathrm{CpG}$ sites that were differentially methylated in association with depressive symptoms [6]. Annotation of these sites implicated genes involved in axon guidance. A study of 150 monozygotic twin pairs discordant for early onset MDD identified 760 differentially methylated $\mathrm{CpG}$ sites, which mapped to neuronal circuitry and plasticity genes [7]. These findings suggest that differences in DNAm may be associated with MDD.

Many lifestyle factors associated with MDD, including smoking [8], obesity [9, 10] and alcohol consumption [11], are associated with differential genome-wide DNAm. These DNAm signatures have been leveraged, using penalised regression to identify a subset of informative $\mathrm{CpG}$ sites, to create DNAm risk scores (MRS), which can predict the trait of interest in an independent cohort. McCartney et al. showed that DNAm scores explained $61 \%$ of the variance in smoking status and $12.5 \%$ of the variance in body mass index (BMI) and alcohol consumption. When modelled alongside PRS, DNAm scores contribute additively to the variance explained for these traits [12]. DNAm therefore acts as an archive of exposure to several risk factors for poor mental health, however the significance of its association with MDD remains unexplored.

A recent study of 581 individuals with depressive symptoms used machine learning methods to train a predictor of MDD using DNAm data. They found that MRS could discriminate future MDD disease status with an area under the curve (AUC) of 0.72 [13]. Notably, this study did not use an independent sample to test their MRS and they discriminated between transient and chronic MDD over a 6year period. The aim of the current study was to use penalised regression to train a predictor of MDD based on DNAm in a large sample using the Generation Scotland: Scottish Family Health Study (GS:SFHS) cohort [14, 15]. A training set of 1223 MDD cases and 1824 controls was used to create an MDD MRS which was then tested in 1970 independent individuals (363 prevalent and 190 incident MDD cases; 1417 controls). As smoking has been consistently associated with differential DNAm [8, 12], we created an MDD MRS that excluded smoking signals (MRS-ns) by training the predictor on a subset of individuals who had never smoked (534 MDD cases and 1017 controls). Using longitudinal clinical data, we also tested whether MRS and MRS-ns derived from blood taken at the baseline assessment would predict future (incident) MDD status at follow-up between 4 and 10 years later. To explore whether the MDD MRS and MRS-ns capture exposure to lifestyle factors associated with MDD, we also tested the association between MDD MRS and MRS-ns, and alcohol use, BMI, smoking status, and pack years, as well as selfreported antidepressant use.

To determine whether the MRS was capturing genetic or environmental liability to MDD, we performed variance component analysis to estimate the single nucleotide polymorphism (SNP)-based and environmental contributions to MDD-associated methylation signatures. Finally, to explore whether the MDD MRS and MRS-ns capture exposure to lifestyle factors associated with MDD, we tested the association between MDD MRS and MRS-ns, and 61 behavioural phenotypes and lifestyle factors. We compared these associations with those observed for PRS that have previously shown association with a wide range of neuropsychiatric traits [16].

\section{Methods}

\section{Study population}

\section{Generation Scotland-the Scottish Family Health Study (GS:SFHS)}

GS:SFHS is a family-based population cohort investigating the genetics of health and disease in $\sim 24,000$ individuals across Scotland [14, 15]. Baseline data were collected between 2006 and 2011. The present study focuses on 5017 individuals for whom DNAm data from a blood draw at baseline contact, baseline phenotypic data and genotype data were available. Environmental data, such as lifestyle factors, were also measured (BMI) or recorded (smoking status and alcohol consumption) on nearly all study participants.

Longitudinal phenotypic data are available for a subset of individuals who responded to a recontact request $[17,18]$. For these individuals we have information on MDD casecontrol status both at baseline and follow-up, which occurred 4-10 years later (2015-2016). GS:SFHS received ethical approval from NHS Tayside Research Ethics Committee (REC reference number 05/S1401/89) and has Research Tissue Bank Status (reference: 15/ES/0040). Written informed consent was obtained from all participants.

\section{Phenotypes}

BMI was calculated using height $(\mathrm{cm})$ and weight $(\mathrm{kg})$ measured by clinical staff during baseline recruitment. 
Alcohol intake was self-reported as part of a pre-clinical questionnaire. Participants were asked whether they were "never", "former" or "current" drinkers. Current drinkers were asked: "During the past week, please record how many units of alcohol you have had".

Smoking status was recorded by asking participants: "Have you ever smoked tobacco?". Answers were recorded as: "Yes, currently smoke; Yes, but stopped within the past 12 months; Yes, but stopped more than 12 months ago; No, never smoked". Previous evidence has shown that cigarette smoking has strong associations with genome-wide DNAm, and effects persist long after smoking cessation, indicating that former smokers may retain DNAm profiles that are similar to current smokers $[10,19]$. Therefore, for the current study, we assigned smoking status as a binary variable, by converting all "Yes" answers to smoker [1], and "No" to non-smoker (0). Using smoking behaviour data, pack years were calculated by multiplying the number of cigarette packs ( 20 cigarettes/pack) smoked per day by the number of years a person has smoked [20].

Antidepressant use was self-reported by participants at the baseline assessment and has been described in greater detail previously ([21]; Supplementary material). See Supplementary Tables 1 and 2 also for demographic differences in lifestyle factors between individuals with an MDD diagnosis and those without one.

Baseline MDD status was measured using the axis-I Structured Clinical Interview of the Diagnostic and Statistical Manual, version IV (SCID) and was administered to participants who answered "yes" to either of two screening questions (see Supplementary materials). MDD status was measured prospectively by remote paper questionnaire between 4 and 10 years after baseline assessment (2015-2016) using the Composite International Diagnostic Interview-Short Form (CIDI-SF) as described previously [17].

Control participants were defined as those individuals who answered "no" to the two screening questions (see Supplementary materials) and did not fulfil criteria for a diagnosis of current or previous MDD following the SCID interview and CIDI-SF remote follow-up assessment. Individuals fulfilling criteria for schizophrenia or bipolar disorder, or who self-reported these diagnoses, were also excluded from both case and control groups.

\section{DNA methylation}

In total, 9873 individuals in GS:SFHS had genome-wide DNAm data profiled from blood samples using the Illumina Human-MethylationEPIC BeadChip. The raw data were acquired, preprocessed and quality checked in two different batches, hereafter named batch $1(n=5190)$ and batch 2 $(n=4588)$.
In batch 1, ShinyMethyl [22] was used to exclude samples where predicted sex mismatched recorded sex, as well as to plot the $\log$ median intensity of methylated and unmethylated signals per array and inspect the output from the control probes; outlying samples detected by visual inspection were excluded. WateRmelon [23] was then used to remove probes in which $>1 \%$ of probes had a detection $p$ value $>0.05$; probes with a beadcount of $<3$ in more than $5 \%$ samples; and probes in which $>5 \%$ of samples had a detection $p$ value $>0.05$ [12]. Multi-dimensional scaling (MDS) plots were inspected to confirm that there were no additional sample outliers. WateRmelon was then used to normalise the data using the dasen method, and lumi [24] was used for conversion to $M$ values, which were then precorrected for relatedness, estimated blood cell types, and processing batch using DISSECT [25], for $\mathrm{CpG}$ sites on autosomal chromosomes. The final dataset comprised corrected $M$ values at 841,753 loci measured for 5087 individuals.

In batch 2, Meffil [26] and ShinyMethyl [22] were used for quality control of the raw data. Using Meffil, samples were removed if: there was a mismatch between selfreported and methylation-predicted sex; they had $>1 \%$ of CpG sites with a detection $p$ value $>0.05$; they showed evidence of dye bias; they were outliers for the bisulphite conversion control probes; and had a median methylated signal intensity $>3$ standard deviations lower than expected. Afterwards, ShinyMethyl was used to perform further quality control, as described above for batch 1. MDS plots were inspected, and outliers were excluded. Meffil was then used again to identify and exclude poor-performing probes, which were deemed as such if: they had a beadcount of $<3$ in $>5 \%$ samples and/or $>5 \%$ samples had a detection $p$ value $>0.05$. The data were normalised using the dasen method in wateRmelon, and the beta2 $\mathrm{m}$ function in lumi [24] was used to generate $M$ values. The final dataset comprised $M$ values for 773,860 loci measured in 4450 individuals.

\section{Genotyping and PRS profiling}

Individuals were genotyped using the Illumina OmniExpress BeadChip. The raw genotype data underwent a series of quality control steps: individuals with a call rate $<98 \%$, SNPs with a genotype rate $<98 \%$, minor allele frequency $<$ $1 \%$, and Hardy-Weinberg $p$ value $<10^{-6}$ were removed from the initial dataset and then imputation was performed using the Sanger Imputation Service with the Haplotype Reference Consortium panel v1.1 [27].

Using the largest available depression GWAS [4], depression PRS were computed using Plink v1.90b4 [28] using SNPs that met a significance level of $p \leq 0.05$, in line with previous studies, which have shown that this threshold 
explains the most variance in MDD status [4]. GWAS summary statistics excluding GS:SFHS were obtained in order to create PRS in the GS:SFHS sample. Clumping was applied using a linkage disequilibrium $r^{2}<0.1$ and a $500-\mathrm{kb}$ window.

\section{DNAm predictor-training and testing datasets}

In order to obtain a training and testing dataset, individuals were separated based on the two batches described above. Supplementary Fig. 1 provides a flowchart summary of the analysis process.

\section{Training dataset}

Batch 1 was used to train two DNAm predictors. The dataset consisted of controls who were screened as unaffected $(n=1824)$ at both baseline and follow-up (i.e., answered "no" to screening questions at baseline and follow-up), or who screened positive but were subsequently found not to fulfil diagnostic criteria for MDD using the SCID. MDD cases were those who screened positive for depression by answering yes to one or more brief screening questions and who subsequently fulfilled criteria for MDD at baseline SCID interview $(n=1223)$. The non-smoker dataset was created by excluding those individuals who had a smoking history from the entire batch 1 dataset ( $N$ excluded $=1496$; i.e. answered "yes" to the question "have you ever smoked tobacco?"). As such, this dataset contained 1017 controls who were screened as unaffected at both baseline and follow-up and 534 MDD cases who were screened positive for depression who answered "no, never smoked" to the question "have you ever smoked tobacco?".

$\mathrm{CpG}$ sites measured in the individuals mentioned above were included as independent variables in a least absolute shrinkage and selection operator (LASSO) penalised regression model described below. Depression status was regressed on age, sex and ten genetic principal components, and the extracted residuals from this model were input as the dependent variable in the LASSO regression model.

LASSO penalised regression models were run using the "glmnet" function in R in order to train DNAm predictors. We applied tenfold cross-validation and the mixing parameter was set to 1 for our LASSO penalty.

\section{Testing dataset}

Batch 2 was used in order to create MDD MRS using the $\mathrm{CpG}$ sites identified in the training set using LASSO regression models. Using the set of $\mathrm{CpG}$ sites selected from the penalised regression, MRS were calculated in the testing dataset using the weights estimated in the training set, first for prevalent depression (Total $n=1780$; cases $=363$; controls $=1417$ ) and then for incident depression (Total $n=1607$; cases $=190$; controls $=1417)$. Prevalent depression refers to those individuals who were depressed at both baseline and follow-up, while incident depression refers to those individuals who were well at baseline but went on to develop MDD.

\section{Statistical methods}

All analyses were conducted using $\mathrm{R}$ (version 3.2.3) in a Linux environment. The $\mathrm{R}$ code for the current analyses is available in Supplementary materials.

\section{Association of MRS and MRS-ns with depression}

The association between both MDD MRS and MDD status was assessed using logistic regression. We tested the association between MRS and prevalent depression (Total $n=$ 1780; cases $=363$; controls $=1417$ ) and between MRS and incident depression (Total $n=1607$; cases $=190$; controls $=1417)$. We repeated these analyses using the MRS-ns score and also performed sensitivity analyses by selecting individuals who had self-reported antidepressant use $\left(\right.$ Total $N_{\text {Prevalent }}=1250$, cases $=198$, controls $=1052$; Total $\quad N_{\text {Incident }}=1195, \quad$ cases $=143, \quad$ controls $=1052$ ). McFadden's $R^{2}$ were calculated to determine the amount of variance in MDD explained by MRS.

We tested whether lifestyle factors previously shown to be associated with both MDD and DNAm [8-12] were associated with the MRS. Using linear regression, we tested whether MRS and MRS-ns were associated with BMI, pack years and alcohol consumption. Logistic regression models were used to test whether MRS and MRS-ns were associated with self-reported antidepressant use and smoking status. To estimate how much variance MRS and MRS-ns explain in MDD status when adjusting for lifestyle factors, MDD status was modelled as a dependent variable with alcohol consumption, BMI, smoking and pack years fit as covariates. We also tested the effect of fitting self-reported antidepressant use in our models to determine whether the MRS and MRS-ns would still significantly contribute to the risk for MDD. This was carried out for both incident and prevalent cases.

In addition, using the "ROCR" $\mathrm{R}$ package, we plotted the predictive ability of MRS and MRS-ns in both incident and prevalent cases and controls using a Receiver Operating Characteristic (ROC) curve, representing the sensitivity and specificity of the score in relation to depression.

\section{Mediation analysis}

Mediation analysis was carried out to illustrate the relationship between PRS, MRS and MDD. In two separate 
mediation models, PRS was set as the independent variable, MRS or MRS-ns as the mediator, and MDD as the outcome. All variables were adjusted for age and sex. In addition, PRS and MRS/MRS-ns were adjusted for the first ten genetic principal components, and MRS/MRS-ns were adjusted for BMI, alcohol consumption and smoking status.

In addition, two mediation models were tested in order to illustrate the relationship between the MRS, smoking and MDD. In the first model, MDD was set as the independent variable, smoking as the mediator and the MRS as the outcome. MDD and smoking were adjusted for age and sex. MRS was adjusted for age, sex, batch and the first ten genetic principal components. In the second model, smoking was set as the independent variable, MRS as the mediator and MDD as the outcome. Covariates for the three variables were consistent with the first mediation model.

The 'lavvan' package in $\mathrm{R}$ was used for mediation analyses [29]. Categorical variables such as sex were transformed into numeric. All variables including covariates were scaled to a mean of 0 and a standard deviation of 1 .

\section{Heritability and environmental contributions to MRS and identification of mQTLs}

A recent study using 5101 individuals from the present GS: SFHS cohort determined the relative genetic and environmental contributions to DNAm at each $\mathrm{CpG}$ site on the EPIC illumina array [5]. This study used a five-component variance component method [30], which estimates the SNP $(G)$ and additional genetic (kinship) $(K)$ contributions to methylation variance alongside contributions from the nuclear family $(F)$, couple $(C)$ and sibling $(S)$ shared environments. Using this approach, we tested the genetic and environmental contributions to the total MRS/MRS-ns using the GKFCS method [30]. Briefly, this decomposes variation in the MRS into the two genetic components $(G$ and $K$ ) and three environmental components (C, F and $\mathrm{S}$ ) using GCTA software [30]. Using a backward-stepwise selection model, we initially fit all five components and then dropped components if they were not significant using Wald or likelihood ratio tests (LRT). This process was repeated until all components significantly contributed to variance in the MRS. This method and the construction of the GKFCS matrices has been previously described in more detail in Xia et al. (2016) [30] and Zeng et al. [5]. We also assessed whether SNPs associated with methylation (mQTLs) at the CpG sites which comprise the MRS were enriched for association with MDD. mQTLs for MRS $\mathrm{CpG}$ sites were identified using the ARIES dataset using the midlife timepoint (http://www.mqtldb.org/) [31]. Significant mQTLs were then tested for their association with MDD by performing a look-up of their $p$ values from summary statistics of the largest GWAS of MDD to date [4]. False discovery rate (FDR) correction was then performed on the MDD $p$ values to identify those mQTLs significantly associated with both $\mathrm{CpG}$ methylation and MDD status after correction for multiple testing.

\section{MRS and PRS associations with an archive of 61 behavioural variables}

The GLM function in $\mathrm{R}$ was used to test associations between the MRS and MDD PRS, which were set as predictor variables, and 61 behavioural, cognitive and lifestyle phenotypes, which were included as outcome variables (for a list of all phenotypes included in the analysis, please see Supplementary Table 3). Covariates in all models included age, sex and ten genetic principal components. FDR correction was applied over all tests (61 outcome variables $\times$ $\mathrm{MRS}+\mathrm{PRS})$ using the p.adjust function in $\mathrm{R}(q<0.05)$.

\section{Pathway analysis}

To annotate $\mathrm{CpG}$ sites comprising the MRS, we used the Infinium MethylationEPIC BeadChip database, which provides information concerning genes, chromosome location, start and end site and other characteristics (https://emea. support.illumina.com/array/array_kits/infinium-methyla tionepic-beadchip-kit/downloads.html). We then used the Functional Mapping and Annotation of Genome-wide association studies (FUMA; 32) in order to identify biological pathways that are associated with the annotated genes (see Supplementary Excel Files $1 \mathrm{~A}$ and $1 \mathrm{~B}$ for a list of $\mathrm{CpG}$ sites and annotated genes for MRS and MRS-ns, respectively). The database tests whether genes of interest are overrepresented in any pre-defined gene sets across a number of databases. We interrogated a number of gene sets using data from the Gene Ontology (GO) Consortium using the FUMA online tool [32].

\section{Results}

One hundred and ninety-six $\mathrm{CpG}$ sites were selected for the MRS predictor that corresponded to the minimum mean cross-validated error within the entire training dataset $(n=$ 3047). Similarly, $144 \mathrm{CpG}$ sites were included in the nonsmoker training dataset $(n=1,551)$ (see Supplementary Excel Files 2A and 2B for a list of $\mathrm{CpG}$ sites and their regression weights for the MRS and MRS-ns, respectively).

\section{Association of MRS with depression}

The MRS was significantly associated with both prevalent (Total $n=1780 ;$ cases $=363$, controls $=1417 ; \beta=0.338$, 
Fig. 1 Prediction of MDD casecontrol status. a Receiver Operating Characteristic (ROC) curve indicating the sensitivity ( $y$-axis) and specificity ( $x$-axis) of methylation risk score (MRS) and methylation risk score trained on non-smokers (MRSns) for both prevalent and incident MDD. The AUC estimates are indicated for each predictor in the legend.

b Variance in prevalent MDD (indicated by $R^{2}(\%)$ on the y-axis) explained by MRS and PRS alone when fitting MDD as the outcome variable and fitting age, sex and ten genetics principal components as covariates. MRS and PRS are then fit in the same model (PRS + MRS) to show their additive contribution to variance explained in MDD. a
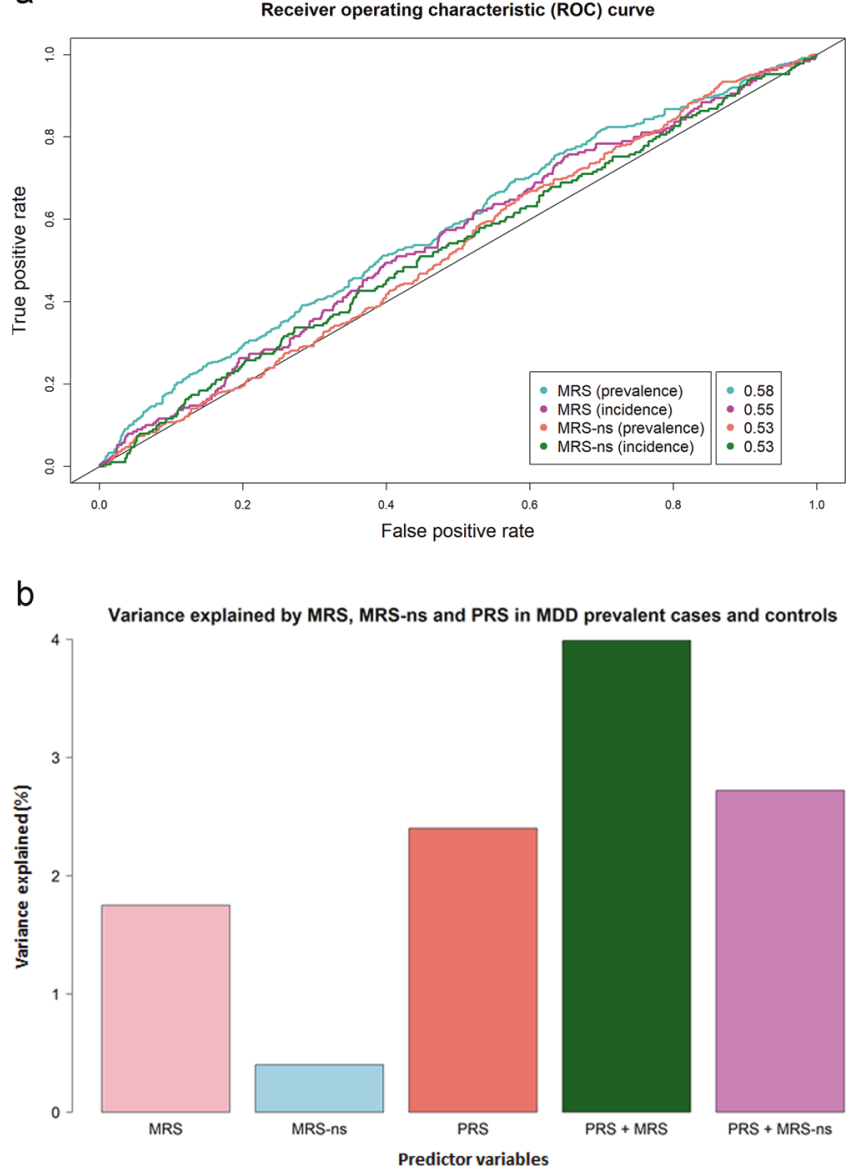

Both MRS $\left(\beta=0.338, p=1.17 \times 10^{-7}, R^{2}=1.75 \%\right)$ and PRS $\left(\beta=0.397, p=1.02 \times 10^{-9}, R^{2}=2.40 \%\right)$ accounted for a small proportion of the variance in risk of prevalent MDD. The model including both $\operatorname{MRS}(\beta=0.327, p=$ $\left.5.66 \times 10^{-7}\right)$ and $\operatorname{PRS} \quad\left(\beta=0.384, \quad p=4.69 \times 10^{-9}\right)$ demonstrated that these two risk scores act additively $\left(R^{2}=\right.$ $3.99 \%$ ) and we found no evidence of an interaction $(\beta=-0.009, p=0.892)$ (Supplementary Table 4). The model including both MRS-ns $(\beta=0.142, p=0.032)$ and PRS $\left(\beta=0.394, p=1.39 \times 10^{-9}\right)$ also found an additive effect of both scores $\left(R^{2}=2.72 \%\right)$ with no evidence of an interaction $(\beta=0.049, p=0.483)$. Figure $1 \mathrm{~b}$ shows the variance in MDD explained (\%) by MRS, MRS-ns and PRS.

We performed sensitivity analyses using MDD cases and controls with no self-reported antidepressant use (Total $N_{\text {Prevalent }}=1250$, cases $=198$, controls $=1052$; Total $N_{\text {Incident }}=1195$, cases $=143$, controls $\left.=1052\right)$, MRS was significantly associated with prevalent $(\beta=0.331, p=$ $\left.6.19 \times 10^{-5}, R^{2}=1.66 \%\right)$ and incident $(\beta=0.232, p=$ $0.011, R^{2}=0.76 \%$ ) MDD. The variance explained in the antidepressant-free subset was slightly lower compared with the full prevalent case-control sample (antidepressantdepression. 
free sample: $R^{2}=1.66 \%$; full sample: $R^{2}=1.75 \%$ ). MRSns was significantly associated with prevalent MDD with no antidepressant use $\left(\beta=0.189, p=0.026, R^{2}=0.507 \%\right)$.

\section{Association of MRS and MRS-ns with lifestyle factors and self-reported antidepressant use}

The MRS was associated with smoking status $(\beta=0.440, p \leq$ $\left.2 \times 10^{-16}, R^{2}=3.2 \%\right)$, pack years $\left(\beta=0.246, p \leq 2 \times 10^{-16}\right.$, $\left.R^{2}=6.5 \%\right)$, alcohol consumption $(\beta=0.092, p=9.85 \times$ $\left.10^{-5}, R^{2}=0.7 \%\right)$ and self-reported antidepressant use $(\beta=$ $0.289, p=0.002, R^{2}=1.1 \%$ ). When partitioning smokers into current and former smokers, the MRS was associated with both (current smokers vs controls: $\beta=1.096, p \leq 2 \times$ $10^{-16}, R^{2}=15.53 \%$; former smokers vs controls: $\beta=0.262$, $\left.p=4.41 \times 10^{-6}, R^{2}=1.06 \%\right)$. BMI was not significantly associated with MRS $\left(\beta=0.039, p=0.099, R^{2}=0.097 \%\right)$ (Supplementary Table 5; Supplementary Fig. 2).

The MRS-ns (trained on non-smokers) was also associated with smoking status $\left(\beta=0.102, p=0.035, R^{2}=\right.$ $0.22 \%)$ and pack years $\left(\beta=0.055, p=0.014, R^{2}=\right.$ $0.27 \%$ ) in an independent dataset, but the strength of association was attenuated compared with the original MRS. The MRS-ns was still associated with smoking status using current smokers only as cases $(\beta=0.256$, $\left.p=0.002, R^{2}=0.97 \%\right)$. MRS-ns was not associated with former smoking when these individuals were compared with controls $\left(\beta=0.059, p=0.264, R^{2}=0.092 \%\right)$. MRSns showed a stronger association with BMI $(\beta=0.053$, $\left.p=0.021, R^{2}=0.246 \%\right)$ than the MRS $(\beta=0.039, p=$ $\left.0.099, R^{2}=0.097 \%\right)$. Alcohol consumption $(\beta=0.024$, $\left.p=0.289, R^{2}=0.01 \%\right)$ and self-reported antidepressant use $\left(\beta=0.084, p=0.365, R^{2}=0.096 \%\right)$ were not associated with MRS-ns (Supplementary Table 5; Supplementary Fig. 3).

\section{Association of MRS and MRS-ns with depression when adjusting for lifestyle factors}

MRS was tested for its association with prevalent and incident depression while adjusting for BMI, alcohol use, smoking status and pack years (lifestyle factors) to determine if any independent contribution remained from the MRS (Table 1 and Fig. 2a). MRS was still associated with prevalent MDD status after adjusting for lifestyle factors $(\beta=0.219, p=0.001)$ but only explained $0.68 \%$ of the variance (compared with $R^{2}=1.75 \%$ in the unadjusted model). For incident depression cases, the MRS was no longer associated with MDD status after adjusting for lifestyle factors (variance explained decreased from $0.52 \%$ prior to adjustment to $0.25 \%$ after adjustment).
Table 1 and Fig. $2 b$ detail the results for the MRS-ns associations. MRS-ns was not associated with prevalent MDD status after adjusting for lifestyle factors $(\beta=0.116$, $p=0.08, R^{2}=0.227 \%$ ).

\section{Mediation analysis}

There was no evidence of mediation or interaction effects of MRS on the relationship between PRS and MDD (Supplementary Figs. 4 and 5).

Smoking significantly mediated the association between MDD and MRS $(\beta=0.071, p<0.001, \mathrm{CFI}=0.976$, TLI $=$ 0.954 , RMSEA $=0.017$ ), with $52.2 \%$ of the mediation taking place through this lifestyle factor (direct association between MDD and MRS before and after adding smoking as the mediator: $C=0.136, C^{\prime}=0.065$ ).

The MRS mediated the association between smoking and MDD $(\beta=0.019, p=0.008, C F I=0.977, T L I=0.995$, RMSEA $=0.017$ ). A smaller proportion of variance of $8.51 \%$ was mediated by the MRS (direct association between smoking and MDD before and after adding MRS as the mediator: $C=0.233, C^{\prime}=0.214$ ).

\section{Heritability and environmental contributions to MRS}

Zeng et al. reported that SNP genetic effects $(\mathrm{G})$ explained 9.5\% of the variance in $\mathrm{CpG}$ methylation across the genome and the additional pedigree effects accounted for $7.2 \%$ of the variance [5]. They found little contribution for the shared environment influencing methylation status. We found significant genetic contributions to the MRS total scores $(G=$ 0.22 [S.E. $=0.07]), K=0.19$ [S.E. $=0.09]$ ), and also significant contributions from the shared couple environment $1(C=0.16$ [S.E. $=0.06])$, but not from the shared sibling environment $\left(S=1 \times 10^{-7}[\mathrm{~S} . \mathrm{E}=0.03]\right)$. A similar pattern was observed for the MRS-ns where genetic effects contributed to a proportion of the observed variance $(G=0.22$, S. $\mathrm{E}=0.07 ; K=0.19, \mathrm{~S} . \mathrm{E}=0.08)$. The recent shared couple environment explained $15 \%$ of the variance in the MRS-ns (S. $\mathrm{E}=0.06$ ), while the shared sibling environment explained only $6 \%$ of the variance in the MRS-ns (S.E. $=0.03$ ).

Methylation quantitative trait loci (mQTLs) were identified for each $\mathrm{CpG}$ comprising the MRS using the ARIES dataset mQTLdb [31] using the middle-age timepoint. Seventy-one of the $196 \mathrm{CpG}$ sites had mQTLs (9740 mQTLs in total). We then tested their association with MDD using summary statistics from the largest GWAS of MDD [4]. MDD GWAS data were available for $8327 / 9740$ mQTL. After FDR correction 536 mQTL were significantly associated with MDD and these spanned $11 \mathrm{CpG}$ sites (Supplementary Table 6). 
Table 1 Association between four lifestyle factors (BMI, smoking status, pack years, alcohol units), MRS, MRS-ns, and prevalent and incident MDD.

\begin{tabular}{lcllllclll}
\hline MRS & \multicolumn{1}{c}{ MRS-ns } \\
\hline Prevalent MDD & Effect size & SE & $p$ value & $R^{2}(\%)$ & Prevalent MDD & Effect size & SE & $p$ value & $R^{2}(\%)$ \\
Body mass index & 0.256 & 0.061 & $2.16 \times 10^{-5}$ & 1.36 & Body mass index & 0.256 & 0.061 & $2.82 \times 10^{-5}$ & 1.36 \\
Smoking status & 0.369 & 0.154 & 0.017 & 2.13 & Smoking status & 0.406 & 0.153 & 0.008 & 2.13 \\
Pack years & 0.239 & 0.074 & 0.001 & 1.003 & Pack years & 0.279 & 0.073 & 0.0001 & 1.003 \\
Alcohol units & 0.08 & 0.066 & 0.232 & 0.13 & Alcohol units & 0.092 & 0.066 & 0.162 & 0.13 \\
MRS & 0.219 & 0.067 & 0.001 & 0.68 & MRS-ns & 0.116 & 0.066 & 0.08 & 0.227 \\
Incident MDD & Effect size & SE & $p$ value & $R^{2}(\%)$ & Incident MDD & Effect size & SE & $p$ value & $R^{2}(\%)$ \\
Body mass index & 0.138 & 0.076 & 0.07 & 0.45 & Body Mass Index & 0.136 & 0.076 & 0.076 \\
Smoking status & 0.629 & 0.19 & 0.0009 & 1.5 & Smoking status & 0.642 & 0.189 & 0.0007 & 0.45 \\
Pack years & -0.0003 & 0.099 & 0.997 & 0.005 & Pack years & 0.026 & 0.098 & 0.794 \\
Alcohol units & -0.109 & 0.095 & 0.248 & 0.11 & Alcohol units & -0.105 & 0.094 & 0.268 \\
MRS & 0.136 & 0.083 & 0.1 & 0.25 & MRS-ns & 0.085 & 0.081 & 0.294 & 0.11 \\
\hline
\end{tabular}

Results presented are those taken from models fitting all lifestyle factors alongside the two MRS in separate models. Effect sizes represent standardised betas. $R^{2}$ represents the variance explained in prevalent and incident MDD by each of the predictor variables.

$S E$ standard error, $M R S$ methylation risk score, $M R S$ - $n s$ methylation risk score trained on non-smokers.

Fig. 2 a Variance in MDD (indicated by $R^{2}(\%)$ on the $y$ axis) explained by four lifestyle factors and MRS. b Variance in MDD (indicated by $R^{2}(\%)$ on the $y$-axis) explained by four lifestyle factors and MRS-ns. Lifestyle factors $=\mathrm{BMI}$, alcohol consumption, smoking status and pack years. Light and dark pink bars indicate the additive variance explained by all lifestyle factors combined in incident (I) and prevalent (P) MDD; the light and dark green bars indicate the additive variance explained by all lifestyle factors with the addition of the MRS to the model.
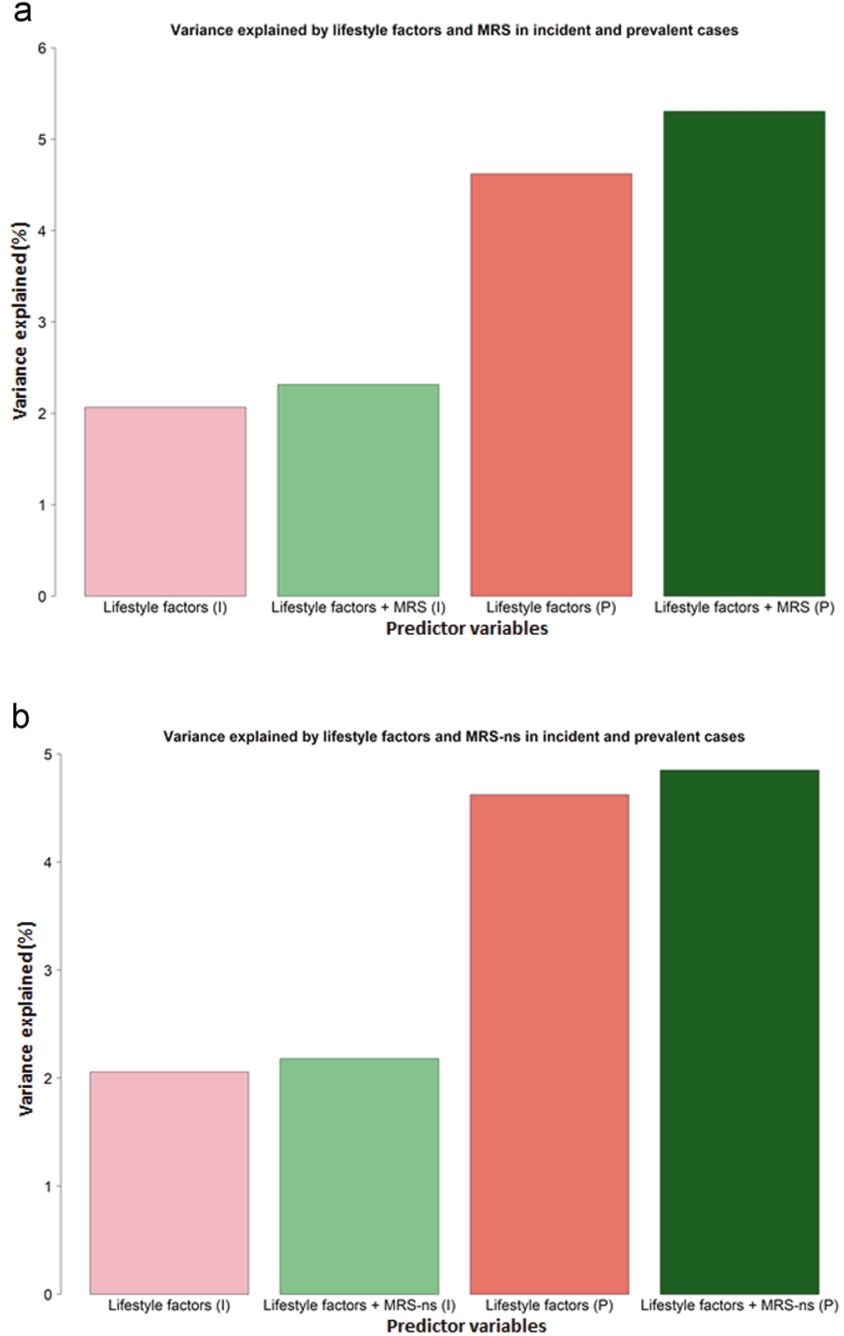
Fig. 3 Phenotypic associations with MRS and PRS.

Associations between mental health, sociodemographic,

lifestyle, physical and cognitive measures and methylation risk score (MRS) in red and polygenic risk score (PRS) in blue; the $x$-axis represents the standardised effect size for each outcome variable listed on the $y$-axis. Error bars represent standard errors of the effect size.

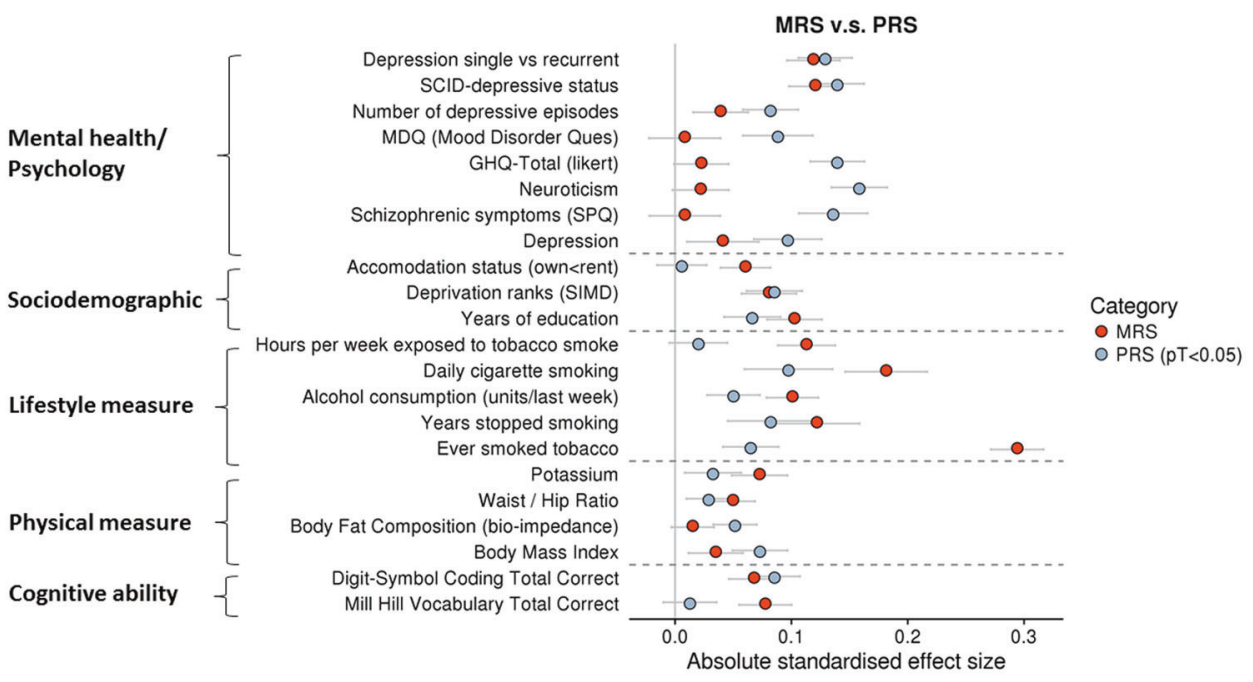

\section{MRS and PRS associations with an archive of 61 behavioural variables}

Of 61 variables investigated, we found 8 phenotypes (categories included cognition, lifestyle, physical and sociodemographic measures) associated with the MRS and 10 phenotypes (categories included cognition, lifestyle, sociodemographic, mental health, physical and disease measures) associated with the PRS. Supplementary Table 7 details the results, including standardised effect size, $p$ value and p-corrected value for each outcome variable. Figure 3 depicts the absolute effect size for significant outcome variables for MRS compared with PRS. Supplementary Fig. 6 depicts the relationship between MRS and MRS-ns and several outcome variables.

\section{Pathway analysis}

We annotated 159 genes to the $196 \mathrm{CpG}$ sites within the MRS, and 111 genes to the $144 \mathrm{CpG}$ sites within the MRSns and used FUMA to identify GO Consortium gene sets enriched for these genes. See Supplementary materials Figs. 7-11 for the GO gene sets, participating genes annotated to the two risk scores, and their respective $p$ value for each gene set, for both MRS and MRS-ns.

Sixty-two significant putative gene sets $\left(P_{\text {corrected }}<0.05\right)$ were identified from the MRS. Of these, 55 were biological processes, of which most included regulation of cellular and molecular processes. Only one of these processes was located in the nervous system: go neurogenesis, involving the generation of cells within the nervous system.

Seventy-two significant putative gene sets $\left(P_{\text {corrected }}<0.05\right)$ were enriched for the MRS-ns genes. Ten of these gene sets involved biological processes occurring in the brain and nervous system and include neurogenesis, neuron differentiation, neuron projection guidance, dopaminergic neuron differentiation, central nervous system development and forebrain development. Fourteen cellular components were identified, eight of which were located in the nervous system and included the following GO gene sets: neuron part, synapse, neuron projection, axon initial segment, paranode region of axon and node of Ranvier. The full lists of biological and cellular components for the MRS and MRS-ns can be found in Supplementary materials (Figs. 7-11).

Both the biological processes and cellular components identified indicate that the MRS-ns is enriched for annotated genes involved in neurodevelopment across multiple areas within the brain, whereas the genes annotated to the MRS have more broad biological functions not specific to the nervous system.

\section{Discussion}

In the current study, we created a methylation risk score for MDD and investigated its association with prevalent depression (individuals who were depressed at both baseline and follow-up) and whether altered DNAm at baseline predicted incident depression between 4 and 10 years later. Our MRS explained $1.75 \%$ of the variance in prevalent MDD compared with $2.40 \%$ of the variance explained by a PRS; additively, the PRS and MRS accounted for 3.99\% of variance explained in total. Although the PRS still outperforms the MRS on predictive ability, it is worth noting that PRS were trained on a sample of 807,579 individuals and the MRS on only 3047 individuals; although the proportion of variance explained by the MRS is currently small, the accuracy and clinical potential of MRS will likely increase as methylation data become more widely available. Therefore, MRS may yet provide clinically valuable information 
about the risk of future MDD. We found that MRS were associated with incident MDD, although they explained less of the variance in future MDD status $(0.52 \%)$.

The MRS was associated with smoking status, pack years and alcohol consumption, suggesting that the MRS may reflect exposure to risky lifestyles known to be associated with MDD. After adjustment for lifestyle factors, the MRS association with MDD was substantially attenuated. These lifestyle factors have previously been associated with MDD [33-37] and are known to robustly associate with patterns of DNAm [12]. The attenuation of the association between MRS and MDD suggests that the DNAm-based predictor of MDD may be acting as a quantifiable archive of the longitudinal effects of these exposures, and other, lifestyle variables. Mediation analyses showed smoking significantly mediated the association between MDD and MRS, with $52.2 \%$ of the relationship being mediated by this lifestyle factor. This result is in line with other findings [8, 38], which indicate a strong influence of smoking on DNAm. In addition, the MRS was also significantly associated with self-reported antidepressant use, although this association does not account for the MRS-MDD associations reported. This finding suggests that MRS may also be sensitive to the effects of antidepressant use and that future studies should examine whether MRS trained on antidepressant use may be valuable as a measure of antidepressant absorption or pharmacological action.

Given the strong association between methylation status and smoking, we re-trained our MRS on a subset of cases and controls who had never smoked. The MRS-ns was still associated with MDD in the testing dataset but did explain less of the variance compared with the MRS $(1.75 \%$ vs $0.4 \%$ ). Surprisingly, the MRS-ns was still associated with smoking status and pack years but to a much lesser extent than the MRS. The MRS explained 3.2\% of the variance in smoking status whereas the MRS-ns only explained $0.22 \%$ of the variance. Interestingly, the MRS-ns was now associated with BMI, a pattern not observed for the MRS. This suggests that when excluding the smoking signals from our dataset, the methylation differences between cases and controls were linked to BMI rather than smoking.

Correction for smoking status in case-control DNAm studies of other traits is an evolving area of methodological development. Correcting for current status alone tends to group together previous smokers and lifelong non-smokers, who may differ significantly in their smoking associated DNAm marks. Future studies may wish to correct for epigenetic smoking measures, as these are more stable and capture a larger proportion of smoking associated DNAm differences [39].

Although MRS were associated with exposure to environmental lifestyle factors, genetic effects are known to influence variation in $\mathrm{CpG}$ methylation status. Zeng et al. [5] recently found that SNP genetic effects explain, on average, 9.5\% of the variance in methylation status at $\mathrm{CpG}$ sites across the genome and $7.2 \%$ of variance could be attributed to pedigree-associated effects. This was highly variable across the genome and only $24,101 \mathrm{CpG}$ sites had statistically significant contributions from SNP genetic effects $(G)$. The $\mathrm{CpG}$ sites comprising the MRS and the MRS-ns had higher contributions from SNP and pedigree-associated genetic effects than expected by chance. The proportion of variance explained by SNP effects for the CpG sites in the MRS was 15.1 and $16.5 \%$ for the MRS-ns. The additional pedigreeassociated effects explained 9.7 and $14.5 \%$ of the variance in methylation for CpG sites making up the MRS and MRS-ns, respectively. Interestingly, $11 \mathrm{CpG}$ sites in the MRS had mQTLs which were also strongly associated with MDD status in GWAS. Although the PRS derived from GWAS appear to be acting additively to the MRS risk for MDD, there are SNPs which associate with both $\mathrm{CpG}$ methylation and MDD risk. Future work should aim to determine whether these MDDassociated genetic variants influence risk for MDD via $\mathrm{CpG}$ methylation at these loci. There was little contribution from the shared family environment; however, when variance component analyses were applied to the total MRS, the shared couple environment significantly contributed to the variance explained. The couples in the GS:SFHS cohort are identified by shared probands and are likely to be co-habiting at the time of recruitment. The couple component therefore represents the recent shared environment and common exposure to lifestyle factors which influence DNAm. We recently showed there are strong couple environmental contributions to smoking and alcohol use which may explain why couples have similar MRS in the present sample [40].

Recent phenome-wide association studies have shown that MDD PRS are associated with a range of psychosocial and mental health phenotypes [16]. Using the same approach, we tested the association between 61 behavioural phenotypes and compared the pattern of association between MRS and PRS. MRS were significantly associated with cognition, lifestyle, physical and sociodemographic variables. In addition to these, PRS were associated with disease and mental health variables, such as MDD, number of depressive episodes and Mood Disorder Questionnaire score. The results indicate that PRS were associated with variables relating to MDD manifestation, as shown in previous studies [16]. Moreover, although both risk scores were associated with sociodemographic measures, such as years of education and deprivation ranks, MRS had a stronger association than PRS, indicating a stronger role played by the MRS in environmental factors [8-12].

Finally, pathway analysis conducted on the genes annotated to the two sets of $\mathrm{CpG}$ sites indicated that MRS annotated genes played a role in regulatory processes, while the MRS-ns annotated genes were enriched in 
neurodevelopmental processes within a large number of areas within the brain. These processes include axon guidance, neurogenesis and neuronal differentiation. This may show that excluding smoking signals from an MDD MRS may lead to uncovering locations along the genome which may play a role in neuronal processes.

The use of a single score instead of thousands of independent loci allows for a more comprehensive analysis investigating the additive effect of a large number of $\mathrm{CpG}$ sites and permits the use of smaller sample sizes. In the current study, we showed that an MRS could discriminate MDD cases and controls with an AUC of 0.58. Using machine learning methods and additional clinical variables, Clark et al. showed that this figure may be increased to 0.74 when investigating recurrent MDD cases only [13]. In this study, we were able to gain insight into a novel association between an MRS and depression, over and above genetic and environmental risk arising from lifestyle factors, as well as examine differences between MRS and PRS in various behavioural phenotypes. However, the MRS has less predictive ability for incident depression, indicating that the score performs better when assessing currently affected individuals. Moreover, although a proportion of the score seems to capture exposure to lifestyle factors, it is unclear what the remainder represents. As such, future studies would benefit from larger sample sizes and longitudinal samples to better understand the contribution of DNAm to MDD risk.

In addition, the diagnostic measures used in the current study differed at baseline (SCID) and follow-up (CIDI-SF). Previous evidence indicates that these two measures do not show perfect agreement [41]. Nevertheless, use of the CIDISF has been well-validated and has good diagnostic accuracy for MDD [42]. Future studies could usefully compare the DNAm profiles of MDD according to different diagnostic instruments.

In conclusion, our results show that an MRS is associated with current and future MDD status, enhancing prediction from PRS and environmental traits. Subsequent to further testing and validation in clinically-ascertained samples, these findings may have future clinical applications for MDD risk stratification and justify further efforts to collect DNAm in larger samples.

Acknowledgements This study is supported by a Wellcome Trust Strategic Award "Stratifying Resilience and Depression Longitudinally" (STRADL) (Reference 104036/Z/14/Z). Generation Scotland received core support from the Chief Scientist Office of the Scottish Government Health Directorates [CZD/16/6] and the Scottish Funding Council [HR03006]. Genotyping of the GS:SFHS samples was carried out by the Genetics Core Laboratory at the Wellcome Trust Clinical Research Facility, Edinburgh, Scotland and was funded by the Medical Research Council UK and the Wellcome Trust (Wellcome Trust Strategic Award (STRADL; Reference as above). DMH is supported by a Sir Henry Wellcome Postdoctoral Fellowship (Reference 213674/Z/18/Z) and a 2018 NARSAD Young Investigator
Grant from the Brain \& Behavior Research Foundation (Ref: 27404). HCW is supported by a JMAS SIM fellowship from the Royal College of Physicians of Edinburgh and by an ESAT College Fellowship from the University of Edinburgh. AMM is additionally supported by Wellcome Trust (104036/Z/14/Z and 216767/Z/19/Z) and UKRI MRC funding (MC PC 17209 and MR/S035818/1). Part of the work was undertaken in The University of Edinburgh Centre for Cognitive Ageing and Cognitive Epidemiology (CCACE), part of the cross council Lifelong Health and Wellbeing Initiative (MR/K026992/1); funding from the Biotechnology and Biological Sciences Research Council (BBSRC) and MRC is gratefully acknowledged. Age UK (The Disconnected Mind project) also provided support for the work undertaken at CCACE. REM is supported by Alzheimer's Research UK major project grant ARUK-PG2017B-10.

\section{Compliance with ethical standards}

Conflict of interest AMM has received grant support from Pfizer, Eli Lilly, Janssen and The Sackler Trust. These sources are not connected to the current investigation. AMM has also received speaker fees from Janssen and Illumina. The remaining authors report no conflicts of interest.

Publisher's note Springer Nature remains neutral with regard to jurisdictional claims in published maps and institutional affiliations.

Open Access This article is licensed under a Creative Commons Attribution 4.0 International License, which permits use, sharing, adaptation, distribution and reproduction in any medium or format, as long as you give appropriate credit to the original author(s) and the source, provide a link to the Creative Commons license, and indicate if changes were made. The images or other third party material in this article are included in the article's Creative Commons license, unless indicated otherwise in a credit line to the material. If material is not included in the article's Creative Commons license and your intended use is not permitted by statutory regulation or exceeds the permitted use, you will need to obtain permission directly from the copyright holder. To view a copy of this license, visit http://creativecommons. org/licenses/by/4.0/.

\section{References}

1. Depression WH. Other common mental disorders: global health estimates. Geneva: World Health Organization; 2017:1-24.

2. Ripke S, Wray NR, Lewis CM, Hamilton SP, Weissman MM, Breen $\mathrm{G}$, et al. A mega-analysis of genome-wide association studies for major depressive disorder. Molecular psychiatry. 2013;18:497-511.

3. Wray NR, Ripke S, Mattheisen M, Trzaskowski M, Byrne EM, Abdellaoui A, et al. Genome-wide association analyses identify 44 risk variants and refine the genetic architecture of major depression. Nat Genet. 2018;50:668.

4. Howard DM, Adams MJ, Clarke TK, Hafferty JD, Gibson J, Shirali M, et al. Genome-wide meta-analysis of depression identifies 102 independent variants and highlights the importance of the prefrontal brain regions. Nat Neurosci. 2019;22:343.

5. Zeng Y, Amador C, Xia C, Marioni R, Sproul D, Walker RM, et al. Parent of origin genetic effects on methylation in humans are common and influence complex trait variation. Nat Commun. 2019;10:1383.

6. Jovanova OS, Nedeljkovic I, Spieler D, Walker RM, Liu C, Luciano $\mathrm{M}$, et al. DNA methylation signatures of depressive 
symptoms in Middle-aged and elderly persons: meta-analysis of multiethnic epigenome-wide studies. JAMA Psychiatry. 2018;75:949-59.

7. Roberson-Nay R, Wolen AR, Lapato DM, Lancaster EE, Webb BT, Verhulst B, et al. Twin study of early-onset major depression finds DNA methylation enrichment for neurodevelopmental genes. bioRxiv. 2018:422345.

8. Joehanes R, Just AC, Marioni RE, Pilling LC, Reynolds LM, Mandaviya PR, et al. Epigenetic signatures of cigarette smoking. Circ Cardiovasc Genet. 2016;9:436-47.

9. Wahl S, Drong A, Lehne B, Loh M, Scott WR, Kunze S, et al. Epigenome-wide association study of body mass index, and the adverse outcomes of adiposity. Nature. 2017;541:81.

10. Mendelson MM, Marioni RE, Joehanes R, Liu C, Hedman ÅK, Aslibekyan S, et al. Association of body mass index with DNA methylation and gene expression in blood cells and relations to cardiometabolic disease: a Mendelian randomization approach. PLoS Med. 2017;14:e1002215.

11. Liu C, Marioni RE, Hedman ÅK, Pfeiffer L, Tsai PC, Reynolds LM, et al. A DNA methylation biomarker of alcohol consumption. Mol Psychiatry. 2018;23:422.

12. McCartney DL, Hillary RF, Stevenson AJ, Ritchie SJ, Walker RM, Zhang Q, et al. Epigenetic prediction of complex traits and death. Genome Biol. 2018;19:136.

13. Clark SL, Hattab MW, Chan RF, Shabalin AA, Han LK, Zhao M, et al. A methylation study of long-term depression risk. Mol Psychiatry. 2019:1-10.

14. Smith BH, Campbell H, Blackwood D, Connell J, Connor M, Deary IJ, et al. Generation Scotland: the Scottish Family Health Study; a new resource for researching genes and heritability. BMC Med Genet. 2006;7:74.

15. Smith BH, Campbell A, Linksted P, Fitzpatrick B, Jackson C, Kerr SM, et al. Cohort Profile: Generation Scotland: Scottish Family Health Study (GS: SFHS). The study, its participants and their potential for genetic research on health and illness. Int $\mathbf{J}$ Epidemiol. 2013;42:689-700.

16. Shen X, Howard DM, Adams MJ, Hill WD, Clarke TK, Deary IJ, et al. A phenome-wide association and Mendelian Randomisation study of polygenic risk for depression in UK Biobank. Nature Communications. 2020;11:1-6.

17. Navrady LB, Wolters MK, MacIntyre DJ, Clarke TK, Campbell $\mathrm{AI}$, Murray AD, et al. Cohort profile: stratifying resilience and depression longitudinally (STRADL): a questionnaire follow-up of Generation Scotland: Scottish Family Health Study (GS: SFHS). Int J Epidemiol. 2017;47:13-4g. 18

18. Habota T, Sandu AL, Waiter G, McNeil C, Steele J, Macfarlane J, et al. Cohort profile for the STratifying Resilience and Depression Longitudinally (STRADL) study: A depression focused investigation of Generation Scotland, using detailed clinical, cognitive, and neuroimaging assessments [version 1; peer review: 1 approved, 1 not approved]. Wellcome Open Res. 2019;4:185.

19. Huan T, Joehanes R, Schurmann C, Schramm K, Pilling LC, Peters MJ, et al. A whole-blood transcriptome meta-analysis identifies gene expression signatures of cigarette smoking. Hum Mol Genet. 2016;25:4611-23.

20. Leffondré K, Abrahamowicz M, Siemiatycki J, Rachet B. Modeling smoking history: a comparison of different approaches. Am J Epidemiol. 2002;156:813-23.

21. Hafferty JD, Campbell AI, Navrady LB, Adams MJ, MacIntyre D, Lawrie SM, et al. Self-reported medication use validated through record linkage to national prescribing data. J Clin Epidemiol. 2018;94:132-42.

22. Fortin JP, Fertig E, Hansen K. shinyMethyl: interactive quality control of Illumina 450k DNA methylation arrays in R. Version 2. F1000Res. 2014;3:175. https://doi.org/10.12688/f1000research. 4680.2.
23. Pidsley R, Wong CC, Volta M, Lunnon K, Mill J, Schalkwyk LC. A data-driven approach to preprocessing Illumina $450 \mathrm{~K}$ methylation array data. BMC Genom. 2013;14:293.

24. Du P, Kibbe WA, Lin SM. lumi: a pipeline for processing Illumina microarray. Bioinformatics. 2008;24:1547-8.

25. Canela-Xandri O, Law A, Gray A, Woolliams JA, Tenesa A. A new tool called DISSECT for analysing large genomic data sets using a Big Data approach. Nat Commun. 2015;6:10162.

26. Min JL, Hemani G, Davey Smith G, Relton C, Suderman M. Meffil: efficient normalization and analysis of very large DNA methylation datasets. Bioinformatics. 2018;34:3983-9.

27. Nagy R, Boutin TS, Marten J, Huffman JE, Kerr SM, Campbell A, et al. Exploration of haplotype research consortium imputation for genome-wide association studies in 20,032 Generation Scotland participants. Genome Med. 2017;9:23.

28. Chang CC, Chow CC, Tellier LC, Vattikuti S, Purcell SM, Lee JJ. Second-generation PLINK: rising to the challenge of larger and richer datasets. Gigascience. 2015;4:7.

29. Rosseel Y. lavaan: An R package for structural equation modeling. J Stat Softw. 2012;48:1-36.

30. Xia C, Amador C, Huffman J, Trochet H, Campbell A, Porteous $\mathrm{D}$, et al. Pedigree-and SNP-associated genetics and recent environment are the major contributors to anthropometric and cardiometabolic trait variation. PLoS Genet. 2016;12:e1005804.

31. Gaunt TR, Shihab HA, Hemani G, Min JL, Woodward G, Lyttleton $\mathrm{O}$, et al. Systematic identification of genetic influences on methylation across the human life course. Genome Biol. 2016;17:61.

32. Watanabe K, Taskesen E, Van Bochoven A, Posthuma D. Functional mapping and annotation of genetic associations with FUMA. Nat Commun. 2017;8:1826.

33. Paperwalla KN, Levin TT, Weiner J, Saravay SM. Smoking and depression. Med Clin N Am. 2004;88:1483-94.

34. De Wit L, Luppino F, van Straten A, Penninx B, Zitman F, Cuijpers P. Depression and obesity: a meta-analysis of community-based studies. Psychiatry Res. 2010;178:230-5.

35. Brière FN, Rohde P, Seeley JR, Klein D, Lewinsohn PM. Comorbidity between major depression and alcohol use disorder from adolescence to adulthood. Compr Psychiatry. 2014;55:526-33.

36. Opel N, Redlich R, Grotegerd D, Dohm K, Heindel W, Kugel H, et al. Obesity and major depression: body-mass index (BMI) is associated with a severe course of disease and specific neurostructural alterations. Psychoneuroendocrinology. 2015;51:219-26.

37. Pedrelli P, Shapero B, Archibald A, Dale C. Alcohol use and depression during adolescence and young adulthood: a summary and interpretation of mixed findings. Curr Addict Rep. 2016; 3:91-7.

38. Lee KW, Pausova Z. Cigarette smoking and DNA methylation. Front Genet. 2013;4:132.

39. McCartney DL, Stevenson AJ, Hillary RF, Walker RM, Bermingham ML, Morris SW, et al. Epigenetic signatures of starting and stopping smoking. EBioMedicine. 2018;37:214-20.

40. Clarke TK, Adams M, Howard DM, Xia C, Davies G, Hayward C, et al. Genetic and shared couple environmental contributions to smoking and alcohol use in the UK population. BioRxiv. 2019, 555961.

41. Wu Y, Levis B, Sun Y, Krishnan A, He C, Riehm KE, et al. Probability of major depression diagnostic classification based on the SCID, CIDI and MINI diagnostic interviews controlling for Hospital Anxiety and Depression Scale-Depression subscale scores: an individual participant data meta-analysis of 73 primary studies. J Psychosom Res. 2020;129:109892.

42. Kessler RC, Andrews G, Mroczek D, Ustun B, Wittchen HU. The World Health Organization composite international diagnostic interview short-form (CIDI-SF). Int J Methods Psychiatr Res. 1998;7:171-85. 